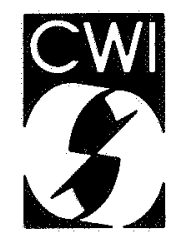

Centrum voor Wiskunde en Informatica Centre for Mathematics and Computer Science

S.M. Verduyn Lunel

Complex analytical methods in RFDE theory 


\title{
Complex Analytical Methods in RFDE Theory
}

\author{
S. M. Verduyn Lunel \\ Centre for Mathematics and Computer Science \\ P.O. Box 4079, 1009 AB Amsterdam, The Netherlands
}

\begin{abstract}
Laplace transformation of a linear autonomous retarded functional differential equation (RFDE) with finite delay yields an analytic equation that has to be solved over a ring of meromorphic functions. This paper presents the technics to study the equation and gives an overview of the recently obtained results related to completeness and convergence of the generalized eigenfunctions of the infinitesimal generator associated to a linear autonomous RFDE.
\end{abstract}

1980 Mathematics Subject Classification : 47D05, 34K05

Key Words \& Phrases : $C_{0}$-semigroups, completeness, exponential series, generalized eigenfunctions, retarded functional differential equations, small solutions, Volterra convolution equation.

Note : This report will be submitted for publication in the proceedings of a meeting on 'Infinite Dimensional Dynamical Systems', Lisbon, May 19-24, 1986

\section{INTRODUCTION}

By applying the Laplace transform to a certain Volterra convolution equation complex function theory can be made to bear on a linear autonomous retarded functional differential equation (RFDE) with finite delay.

In combination with the functional analytical information obtained in $[3,7]$, theorems such as the Paley-Wiener theorem and the Ahlfors-Heins theorem turn out to be quite useful in the study of RFDEs.

Although the matrix equation obtained by Laplace transformation has to be solved over a ring of meromorphic functions, it turns out that information about the exponential growth of the elements involved in the equation is sufficient to prove existence of solutions with certain properties.

This paper will give an overview, detailed results can be found in $[8,9,10]$.

The organization of the paper is as follows. In section 2 we consider the linear autonomous RFDE, give definitions and motivate the basic questions before we start with the Volterra convolution equation in section 3. In section 4 we consider the Laplace transformed version of the Volterra convolution equation and present convergence results for the exponential series expansion of the solution.

\section{THE LINEAR AUTONOMOUS RFDE}

Consider the linear autonomous RFDE with finite delay given by

$$
\begin{aligned}
& \dot{x}(t)=L x_{t}, \\
& x_{0}=\phi, \\
& \phi \in C=C[-h, 0],
\end{aligned}
$$

where $t \geqslant 0, x_{t}=x(t+\cdot)$ on $[-\mathrm{h}, 0]$ and $\mathrm{L}$ is a continuous mapping from $\mathrm{C}$ into $\mathbb{R}^{n}$, and hence is given by

$$
L \phi=\int_{0}^{h} d \zeta(\theta) \phi(-\theta)
$$


where $\zeta$ is a matrix valued function on $\mathbb{R}^{n}$ that is left continuous, of bounded variation and constant on the interval $[h, \infty)$.

Translation along the solution induces a $C_{0}$-semigroup

$$
T(t) \phi=x(t+\cdot ; \phi)=: x_{t}
$$

with infinitesimal generator

$$
A \phi=\dot{\phi},
$$

defined on

$$
\mathscr{D}(A)=\{\phi \in C: \dot{\phi} \in C \text { and } \dot{\phi}(0)=L \phi\} \text {. }
$$

From the explicit formula for the resolvent [7]

$$
R(\lambda, A)=(\lambda I-A)^{-1}
$$

one can deduce that the resolvent is a compact operator. Consequently the spaces

$$
\mathscr{N}\left((\lambda I-A)^{k}\right) \text {, }
$$

$\lambda \in \sigma(A)$, are finite dimensional, become stable for finite $k=m_{\lambda}$, the order of the eigenvalue $\lambda$, and the spectral decomposition

$$
C=\Re\left((\lambda I-A)^{m_{\lambda}}\right) \oplus \mathscr{R}\left((\lambda I-A)^{m_{\lambda}}\right)
$$

holds.

The spectral projection corresponding to the spectral decomposition is given by

$$
P_{\lambda} \phi=\int_{\mathrm{f}_{\lambda}} R(z, A) \phi d z
$$

where $\Gamma_{\lambda}$ is a contour enclosing $\lambda$ but no other point of the discrete set $\sigma(A)$.

Note that, in general, the spectral decomposition is not orthogonal.

Let $\Re_{C}$ denote the linear subspace generated by

$$
\mathscr{x}\left((\lambda I-A)^{m_{\lambda}}\right), \lambda \in \sigma(A) .
$$

The linear subspace $\Re_{C}$ will be called the generalized eigenspace of $A$.

Define the ascent of a semigroup $\{T(t)\}$ by the value

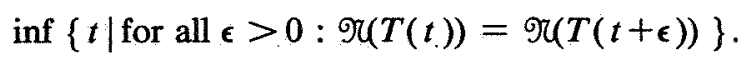

Let $\alpha$ denote the ascent of $\{T(t)\}$ and $\delta$ denote the ascent of the adjoint semigroup $\left\{T^{*}(t)\right\}$.

DEFINITION 2.1. A solution $x$ of equation (2.1) is called a small solution if

$$
\lim _{t \rightarrow \infty} e^{k t} x(t)=0,
$$

for all $k \in \mathbb{R}$.

In 1971 Henry [5] proved the following results 


\section{THEOREM 2.2 .}

(i) $\alpha \leqslant n h$;

(ii) Small solutions are in the nullspace of $T(\alpha)$;

(iii) $\bar{\Re}_{C}=\overline{\Re(T(\delta))}$.

Note that because of (iii), $\delta=0$ implies completeness of the generalized eigenfunctions - i.e. $\overline{\mathscr{T}}_{C}=C$. Also note that $\Re(T(t))$ 'decreases' with increasing time $\mathrm{t}$ because the solution becomes smoother, but that the closure of the range, $\overline{\mathscr{R}(T(t))}$, becomes stable after finite time since, by duality, $\delta \leqslant n h$ holds as well.

The folowing questions were the motivation for a further study of the linear autonomous RFDE.

QUESTION I. Does $\alpha=\delta$ hold?

A related question is : Is completeness equivalent to the absence of non-trivial small solutions?

QUESTION II. Is there an explicit characterization of the ascents $\alpha, \delta$ in terms of the kernel $\zeta$ such that completeness can be verified easily?

From the explicit formula for the resolvent $R(\lambda, A)$ [7] one can show that

$$
\bigcap_{\lambda \in \sigma(A)} \mathcal{R}\left((\lambda I-A)^{m_{\lambda}}\right)=\mathscr{T}(T(\alpha))
$$

Therefore one might ask the following question.

QuEstion III. Can we extend the spectral decomposition over all $\lambda \in \sigma(A)$ to obtain the decomposition

$$
C=\overline{\mathfrak{T}}_{C} \oplus \mathscr{\mathcal { U }}(T(\alpha)) ?
$$

In this paper we will discuss the above questions and we will give an overview of the answers which were recently obtained.

REMARK 2.3. In this paper we use the state space C. However, all our results can be extended to the state space $M_{p}=\mathbb{R}^{n} \times L^{p}[-h, 0]$ introduced by Delfour, Manitius and others [2]. Here we will work in the C-framework because the results of Diekmann [3] make clear that there is no need anymore to extend the state space to $M_{p}$ because there is a natural extension from $\mathrm{C}$ to $M_{\infty}$ which satisfies all our needs for a larger state space. Moreover, the semigroups on $\mathrm{C}$ and $M_{\infty}$ have equal ascents and hence the results obtained for the $\mathrm{C}$-framework can be extended without difficulties to the $M_{\infty}$-framework.

\section{THE RFDE VERSUS THE VOLTERRA CONVOLUTION EQUATION}

The derivative of the solution of the linear autonomous RFDE (2.1) satisfies a renewal equation of the form

$$
\dot{x}-\zeta * \dot{x}=h,
$$

where $\mathrm{h}$ is defined on $\mathbb{R}_{+}$and is constant on the interval $[h, \infty)$.

The convolution product is, as usual, defined by 


$$
h * g(t)=\int_{0}^{t} \zeta(\theta) g(t-\theta) d \theta
$$

where $t \geqslant 0$.

Also, the adjoint semigroup $T^{*}(t)$ can be considered as a semigroup acting on forcing functions in the equation

$$
x-\xi^{T} * x=g,
$$

where $\mathrm{g}$ is defined on $\mathbb{R}_{+}$, left continuous, of bounded variation, and constant on the interval $[h, \infty)$, and $\zeta^{T}$ denotes the transposed kernel.

Formal calculations show that in fact equation (2.1) is equivalent to the Volterra convolution equation

$$
x-\zeta * x=f
$$

where $f$ is an element of $F$, the supremum-normed Banach space of all continuous functions defined on $\mathbb{R}_{+}$, that are constant on the interval $[h, \infty)$.

The equivalence map from an initial condition for (2.1) onto a forcing function for (3.1) is given by

$$
\phi \rightarrow \phi_{-h}-\zeta * \phi_{-h},
$$

and corresponds to shifting the the solution over a distance $h$.

We can rescale the equation (3.1) such that the rescaled kernel $\tilde{\zeta}$ becomes an element of $L^{1}\left(\mathbb{R}_{+}\right)$. A straight forward contraction argument can be used to prove the existence and uniqueness of the rescaled solution $\tilde{x}(\cdot ; f)$ as an element of $C_{0}\left(\mathbb{R}_{+}\right) \cap L^{2}\left(\mathbb{R}_{+}\right)$. The solution $x(\cdot ; f)$ equals $\tilde{x}(\cdot ; f) e^{\gamma t}$ where $\gamma$ is a fixed positive number, the scaling factor. Without loss of generality we may assume $\gamma=0$.

Let $\{S(t)\}$ denote the $C_{0}$-semigroup acting on forcing functions according to the induced action of $\{T(t)\}$ on forcing functions under the equivalence mapping (3.2). Then

$$
S(t) f=x_{t}(\cdot ; f)-\zeta * x_{t}(\cdot ; f)
$$

and $\{T(t)\}$ and $\{S(t)\}$ have equal ascent.

\section{LAPLACE TRANSFORMATION OF THE VOLTERRA CONVOLUTION EQUATION}

Since the solution $x=x(\cdot ; f)$ of the (rescaled) equation $(3.1)$ is an element of $L^{2}\left(\mathbb{R}_{+}\right)$we can Laplace transform the equation (3.1) to obtain for $\operatorname{Re}(z)>0$

$$
\mathcal{E}[x](z)=\Delta^{-1}(z)\left\{f(h)+z \int_{0}^{h} e^{-z t}(f(t)-f(h)) d t\right\}
$$

where $\Delta(z)$, the characteristic matrix of $(3.1)$, denotes the complex matrix valued function

$$
\Delta(z)=z I-\int_{0}^{h} e^{-z \theta} d \zeta(\theta) .
$$

The expression (4.1) yields a meromorphic continuation of $\mathcal{L}[x]$ to the whole complex plane. We will denote this meromorphic continuation by $H(\cdot ; f)$, it is clear that the only singularities of $H(\cdot ; f)$ 
occur at $z=\lambda_{j}$, where $\lambda_{j}$ is a root of the equation

$$
\operatorname{det} \Delta(z)=0 \text {. }
$$

The residues at these poles have the form

$$
\underset{z=\lambda_{j}}{\operatorname{Res}}\left\{e^{z t} H(z ; f)\right\}=p_{j}(t ; f) e^{\lambda_{j} t}
$$

where $p_{j}(\cdot ; f)$ is a polynomial of degree $\left(m_{\lambda}-1\right)$, with $m_{\lambda}$ the multiplicity of the zero $\lambda_{j}$. The series

$$
\sum_{j=0}^{\infty} p_{j}(t ; f) e^{\lambda_{j} t}
$$

is called the exponential series expansion for the solution $x(\cdot ; f)$. Under the equivalence map of section 3 the exponential series expansion for a solution of the Volterra convolution equation is mapped onto the spectral projection series of the corresponding solution of the RFDE (2.1).

DEFINITION 4.1. An entire function $F$ of order 1

$$
\text { i.e. } \limsup _{r \rightarrow \infty} \frac{\log \log M(r)}{\log r}=1 \text {, }
$$

where $M(r)=\max \left\{\left|F\left(r e^{i \theta}\right)\right|: 0 \leqslant \theta \leqslant 2 \pi\right\}$,

is of exponential type $\tau$ if

$$
\limsup _{r \rightarrow \infty} \frac{\log M(r)}{r}=\tau,
$$

where $0 \leqslant \tau \leqslant \infty$.

The exponential type of a vector valued entire function is defined as the maximal exponential type of the components.

If a globally defined meromorphic function is given by the quotient of two entire functions of exponential type, then the exponential growth of the meromorphic function is defined by the difference of the exponential type of the numerator and the denominator.

The following application of the Paley-Wiener theorem and the Ahlfors-Heins theorem, Boas [1], will be the key in the exponential type calculus used in this paper.

THEOREM 4.2. Let $F$ be an entire function which is uniformly bounded in the closed right half plane. Then $F$ is of exponential type $\tau$ and $L^{2}$-integrable along the imaginary axis if and only if

$$
F(z)=\int_{0}^{\tau} e^{-z t} \phi(t) d t
$$

where $\phi \in L^{2}[0, \tau]$ and $\phi$ does not vanish a.e. in some neighborhood of $\tau$. Moreover, the exponential growth in different directions is given by

$$
\limsup _{r \rightarrow \infty} \frac{\log \left|F\left(r e^{i \theta}\right)\right|}{r}=-\tau \cos \theta
$$


for $\theta$ in a dense set of $\left[\frac{1}{2} \pi, \frac{3}{2} \pi\right]$.

Functions of the form (4.4) will be called Paley-Wiener functions. Note that the exponential type of the product of two Paley-Wiener functions equals the sum of the exponential types of the factors. This property makes the exponential type calculus so useful.

Since the entries of $\Delta(\mathrm{z})$ are polynomials of degree 1 with coefficients constants plus Paley-Wiener functions of exponential type less than or equal to $h$, it follows that $\operatorname{det} \Delta(z)$ will be a polynomial of degree $n$ with coefficients constants plus Paley-Wiener functions of exponential type less than or equal to $\mathrm{nh}$.

The same arguments apply to the minors $M_{i j}$ of adj $\Delta(z)$. These will be polynomials of degree (n-1) with coefficients constants plus Paley-Wiener functions of exponential type less than or equal to (n1)h.

Define $\epsilon$ by

$$
\text { exponential type } \operatorname{det} \Delta(z)=n h-\epsilon,
$$

and $\sigma$ by

$$
\max _{i, j} \text { exponential type } M_{i j}=(n-1) h-\sigma .
$$

TheOREM 4.3. If $\epsilon>0$ then $\sigma<\epsilon$.

Proof. Suppose $\sigma=\epsilon$ then

$(n-1)(n h-\epsilon)=\operatorname{type}(\operatorname{det} \Delta(z))^{n-1}=\operatorname{type} \operatorname{det} \operatorname{adj} \Delta(z)$,

and also,

type det adj $\Delta(z) \leqslant n((n-1) h-\epsilon)=(n-1)(n h-\epsilon)-\epsilon$,

which yields a contradiction if $\epsilon>0$.

Equation (4.1) implies that the exponential growth of $H(\cdot ; f)$ has to be less than or equal to $(\epsilon-\sigma)$.

In case of small solutions, i.e. solutions with an entire Laplace transform, it follows that this Laplace transform has exponential type less than or equal to $(\epsilon-\sigma)$. By theorem (4.2)

$$
\int_{0}^{\infty} e^{-z t} x(t) d t=\int_{0}^{\epsilon-\sigma} e^{-z t} x(t) d t
$$

and hence the solution will be zero for $t \geqslant(\epsilon-\sigma)$, and the ascent $\alpha$ of $\{S(t)\}$ will be less than or equal to $(\epsilon-\sigma)$. In fact one can prove equality by constructing a forcing function $f$ such that the right hand side of equation (4.1) is entire and has exponential type equal to $(\epsilon-\sigma)$. This has been done in [8], where we proved the following theorem

THEOREM 4.4. The ascent $\alpha$ of the semigroup $\{S(t)\}$ is given by

$$
\alpha=\epsilon-\sigma .
$$


Since $\epsilon$ and $\sigma$ are invariant under transposing the kernel $\zeta$ we obtain as a corollary of the above theorem.

COROLlary 4.5 .

$$
\alpha=\delta
$$

This means that the first two questions of section 2 are answered positively and that completeness is equivalent to 'exponential type $\operatorname{det} \Delta(z)$ equals nh'.

Another application of the approach introduced above is related to convergence criteria for exponential series expansions.

Let $\Re_{F}$ denote the space of forcing functions $\mathrm{f} \in \mathrm{F}$ such that the solution $x(\cdot ; f)$ can be given by a finite exponential series. Clearly, under the equivalence map of section $3, \mathfrak{R}_{F}$ corresponds to $\Re_{C}$.

The exponential series for a solution can be obtained by applying the inverse Laplace transform theorem to the equation (4.1) and then using the Cauchy theorem of residues. From this, it follows that $C_{0}\left(\mathbb{R}_{+}\right)$-convergence of the exponential series is equivalent to

$$
\left\|\int_{C_{n}} e^{z t} H(z ; f) d z\right\|_{C_{0}\left(\boldsymbol{R}_{+}\right)} \rightarrow 0, \text { as } n \rightarrow \infty
$$

where $C_{n}$ denotes that arc of the circle with radius $\mathrm{n}$ which is contained in the left half plane.

If $H(\cdot ; f)$ has positive exponential growth it is clear that $(4.4)$ will not hold. In case $H(\cdot ; f)$ has no exponential growth we have the following theorem [9].

THEOREM 4.6. Let $f$ be an absolutely continuous element of $F$ such that

$$
\text { exponential type } \operatorname{adj} \Delta(z) z \int_{0}^{\infty} e^{-z t} f(t) d t<\text { exponential type } \operatorname{det} \Delta(z) \text {, }
$$

then the solution $x(\cdot ; f)$ of $(3.1)$ can be given as a $C_{0}\left(\mathbb{R}_{+}\right)$-convergent exponential series.

Due to the fact that the forcing function associated to an element of the $C_{0}\left(\mathbb{R}_{+}\right)$-closure of the span of the exponential series is not necessarely absolutely continuous, we have that an element of the $C_{0}\left(\mathbb{R}_{+}\right)$-closure of the span of the exponential series is not necessarely given by a $C_{0}\left(\mathbb{R}_{+}\right)$-convergent exponential series. However, for absolutely continuous forcing functions the lack of convergence of the exponential series is only in the point $t=0$ as is shown by the following theorem [9].

THEOREM 4.7. The solution $x(\cdot ; f)$ is an element of the $C_{0}\left(\mathbb{R}_{+}\right)$-closure of the span of

$$
\left\{\left(p_{j}(t ; f) e^{\lambda_{j} t}\right)_{j=0}^{\infty}\right\}
$$

if and only if the forcing function $f$ satisfies the condition

$$
\text { exponential type } \operatorname{adj} \Delta(z) z \int_{0}^{\infty} e^{-z t} f(t) d t \leqslant \text { exponential type } \operatorname{det} \Delta(z) .
$$

Moreover, if $f$ is absolutely continuous then the solution $x(\eta+\cdot ; f)$ can be given as a $C_{0}\left(\mathbb{R}_{+}\right)$convergent exponential series for every $\eta>0$.

Since all non-trivial small solutions have a Laplace transform with positive exponential type it is 
clear that a forcing function corresponding to a small solution can not satisfy the condition of Theorem 4.7. Consequently, we have the following corollary

COROLLARY 4.8 There are no small solutions in the $C_{0}\left(\mathbb{R}_{+}\right)$-closure of the span of

$$
\left\{\left(p_{j}(t ; f) e^{\lambda_{j} t}\right)_{j=0}^{\infty}\right\} \text {. }
$$

And because of the equivalence map defined in section 3 .

COROLlary 4.9

$$
\overline{\mathscr{T}}_{C} \cap \Re(T(\alpha))=\{0\}
$$

This corollary shows that $\bar{\pi}_{C}$ is a closed invariant subspace of $C$ and answers a question posed by Hale in his book on functional differential equations [4] positively.

Moreover, corollary (4.9) implies, by duality, that

$$
\overline{\mathfrak{T}}_{C} \oplus \mathscr{T}(T(\alpha))
$$

decomposes at least a dense subspace of $\mathrm{C}$. In general this direct sum is not closed. This is caused by the exceptional behaviour at the point $t=0$ and is related to the lack of convergence in $t=0$ of the spectral projection series. Detailed results will be given in [10].

\section{EXAMPLE}

Although the condition on $\mathrm{f}$ for being an element of $\overline{\mathfrak{N}}_{F}$ are technical, they are rather simple to check in case we are dealing with differential difference equations. It turns out that $\bar{\pi}_{F}$ can be explicitly determined for this type of equations.

\section{EXAMPLE:}

Consider the equation:

$$
\begin{aligned}
& \dot{x}_{1}(t)=-x_{2}(t)+x_{3}(t-1) \\
& \dot{x}_{2}(t)=x_{1}(t-1) \\
& \dot{x}_{3}(t)=0
\end{aligned}
$$

then the characteristic matrix becomes:

$$
\Delta(z)=\left(\begin{array}{ccc}
z & 1 & -e^{-z} \\
-e^{-z} & z & 0 \\
0 & 0 & z
\end{array}\right)
$$

and $\Delta^{-1}(z)$ becomes:

$$
\Delta^{-1}(z)=\left[\begin{array}{ccc}
z^{2} & -z & z e^{-z} \\
z e^{-z} & z^{2} & e^{-2 z} \\
0 & 0 & z^{2}+e^{-z}
\end{array}\right] \frac{1}{z\left(z^{2}+e^{-z}\right)}
$$


Are there any small solutions?

Yes, $\alpha=\epsilon-\sigma=2-0=2>0$.

So no completeness of the generalized eigenfunctions.

Description of the set $\overline{\mathscr{N}}_{F}$ :

Apply $\Delta^{-1}(z)$ to the Laplace transform of $f$ and use Theorem 4.7, the first equation yields no information, the third equation forces $f_{3}$ to be constant, finally the second equation yields:

$$
\overline{\mathscr{T}}_{F}=\left\{f \in F: f_{1}(t)=f_{3}(1) t+c, c \in \mathbb{R} \text {, and } f_{3}(t)=f_{3}(1)\right\} \text {. }
$$

\section{REFERENCES}

[1] R. BoAs, 'Entire Functions,' Academic Press, New York, 1954.

[2] M. C. Delfour AND A. Manitrus, The structural operator $F$ and its role in the theory of retarded systems, I, II, J. Math. Anal. Appl. 73 (1980), 466-490; 74 (1980), 359-381.

[3] O. Diekmann, Perturbed dual semigroups and delay equations, Proceedings of 'Infinite Dimensional Dynamical Systems', Lisbon, 1986

[4] J. K. HALE, 'Theory of Functional Differential Equations,' Springer-Verlag, New York, 1977.

[5] D. HenRY, Small solutions of linear autonomous functional differential equations, $J$. Differential Equations 9 (1971), 55-66.

[6] D. Salamon, 'Control and Observation of Neutral Systems,' Research Notes in Mathematics Vol. 91, Pitman, London, 1984.

[7] S. M. VerduYN LUNel, 'Linear Autonomous Retarded Functional Differential Equations: A sharp version of Henry's theorem,' Report AM-R8405, Centre for Mathematics and Computer Science, Amsterdam.

[8] S. M. VeRdUYN LUNEL, A sharp version of Henry's theorem on small solutions J. Differential Equations 62 (1986), 266-274.

[9] S. M. VerduYN Lunel, Exponential series expansion for solutions of a Volterra equation of the convolution type, to appear.

[10] S. M. VERDUYN LUNEL, An invariant subspace for a linear autonomous RFDE carrying all the information, to appear. 